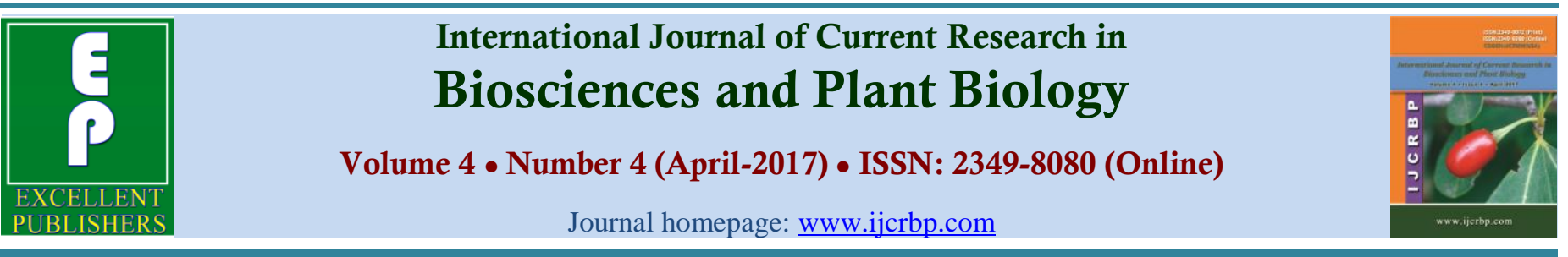

\title{
Cloning and Sequence Analysis of a Flavonol Synthase (FLS2) Gene from Ginkgo biloba
}

\author{
Hui Zeng, Li Yang, Hua Rong, Weiwei Zhang*, Feng Xu and Xin Zhang
}

College of Horticulture and Gardening, Yangtze University, Jingzhou, 434025, China

*Corresponding author.

\begin{tabular}{|c|c|}
\hline Abstract & Article Info \\
\hline \multirow{6}{*}{$\begin{array}{l}\text { Flavonol Synthase, belongs to 2-oxoglutarate-dependent dioxygenase (2-ODD) } \\
\text { superfamily, was involved in flavonol biosynthesis in plants. In this study, we cloned a } \\
\text { cDNA encoding flavonol synthase from Ginkgo biloba (designated as GbFLS2).The } \\
\text { cDNA of GbFLS2 gene is } 1251 \text { bp and contains a } 1017 \text { bp open reading frame encoding } \\
338 \text { amino acids. The deduced protein of GbFLS2 displays extensive homology to FLS } \\
\text { proteins from other plants such as Pinus radiata, Dioscorea alata, Camellia sinensis and } \\
\text { Epimedium sagittatum. Phylogenetic analysis indicated that the GbFLS2 has a closer } \\
\text { relationship with FLS from gymnosperm plants than from other plant species. The results } \\
\text { suggest that GbFLS2 is a member of the 2-ODD superfamily, and it is from the same } \\
\text { ancestor as FLS proteins of other gymnosperm plants. }\end{array}$} & $\begin{array}{l}\text { Accepted: } 15 \text { March } 2017 \\
\text { Available Online: } 06 \text { April } 2017\end{array}$ \\
\hline & Keywords \\
\hline & Flavonol Synthase \\
\hline & Gene clone \\
\hline & Ginkgo biloba \\
\hline & Sequence analysis \\
\hline
\end{tabular}

\section{Introduction}

Flavonoids is a kind of important secondary metabolites widely distributed in plants. Flavonols is one of the major subclasses of flavonoids and has been identified with the antioxidant, antiproliferative, antiangiogenic, and neuropharmacological properties (Kim et al., 2006; Owens et al., 2008). In addition, flavonols have many important physiological functions in plants, such as auxin transport regulation, flower color modulation, UV protection, and signaling (Böhm et al., 1998; Havsteen, 2002; Toh et al., 2013). Flavonol synthase (FLS), a member of the 2-oxoglutarate-dependent dioxygenase (2-ODD) superfamily, plays a major role in the flavonols biosynthesis. So far, many FLS cDNAs have been isolated from different plant species, such as Tartary buckwheat $(\mathrm{Li}$ et al., 2013), Camellia sinensis (Lin et al., 2007), Acacia confusa (Toh et al., 2013), Citrus unshiu (Wellmann et al., 2002), Harosoy (Takahashi et al., 2007), Zea mays (Falcone Ferreyra et al., 2010) and G. biloba (Xu et al., 2012).

Flavonols are synthesized through the flavonoid pathway. In G. biloba, several genes related to the flavonoid pathway, such as GbCHS1 (Pang et al., 2005), GbPAL (Xu et al., 2008), GbF3H (Shen et al., 2006), GbCHS2 (Xu et al., 2007) and GbCHI (Cheng et al., 2011) have been cloned and characterized. To figure out biosynthetic pathway of flavonoids in G. biloba, each gene must be identified and characterized. In a previous study, we also reported the isolation and characterization of a flavonol synthase gene from G. biloba, function analysis indicated that GbFLS is a bifunctional enzyme within the flavonol biosynthetic pathway, recombinant GbFLS1 protein could 
catalyze the formation of dihydrokaempferol to kaempferol and the conversion of kaempferol from naringenin (Xu et al., 2012). In the current study, another flavonol synthase gene was isolated from G. biloba, the cDNA and protein structure was analyzed via bioinformatics methods.

\section{Materials and methods}

\section{Plant material}

The leaves of G. biloba were used for $F L S$ gene clone in this study. 18-year-old grafts G. biloba was grown in the Botanical Garden of Yangtze University, in China. The leaves were collected and frozen in liquid nitrogen, then kept at $-80^{\circ} \mathrm{C}$ prior to RNA extraction.

\section{RNA extraction and Isolation of GbFLS2}

Total RNA was extracted from leaves tissues of G. biloba using the MiniBEST Plant RNA extraction kit (Dalian TaKaRa, China). The cDNA used as template to amplify GbFLS2 gene was obtained from reverse transcribed of total RNA, which was completed by using the PrimeScript ${ }^{\mathrm{TM}} 1$ st Strand cDNA Synthesis Kit (TaKaRa, Dalian, China). A pair of specific primers $G b F L S 2 F$ ( $5^{\prime}$ - TGAACACCAACTGATCTGAATCGTA-3') and GbFLS2R ( $5^{\prime}$-CCAATACACATTTATAAAAGACTTGGTAGG-3') were designed based on transcriptome sequence, and synthesized by Shanghai Sangon Biotechnology company (In China). PCR reaction was conducted under the following conditions: denaturation at $94^{\circ} \mathrm{C}$ for 4 min; followed by 32 cycles of $94^{\circ} \mathrm{C}$ for $30 \mathrm{~s}, 60^{\circ} \mathrm{C}$ for 30 sand $72^{\circ} \mathrm{C}$ for $90 \mathrm{~s}$; extension at $72^{\circ} \mathrm{C}$ for $10 \mathrm{~min}$. The purified PCR product was ligated into pMD18-T cloning vector (Dalian TaKaRa, China) according to the manufacturer's instructions. Then the pMD18-T vector was transformed into E. coli TOP10 competent cells. M13 universal primers were used to screen cloned DNA fragments, and the positive clones were sequenced by Shanghai Sangon Biotechnology Company.

\section{Bioinformatics analysis}

The obtained nucleotide sequence and deduced amino acid sequence were compared through database search using online bioinformatics tools (NCBI, http://www.ncbi.nlm.nih.gov/BLAST/). Software Vector NTI Suite V 11.5 and DNAMAN8 was used to analyze cDNA sequence of FLS gene. Physical and chemical parameters of GbFLS2 protein were analysed using ExPASy (http://www.expasy.org/). The secondary structure of GbFLS2 protein was analyzed by SOPMA tool. GbFLS2 and other FLS proteins obtained from GenBank were aligned through the program Align X (Vector NTI Suite V 11.5). Phylogenetic tree of FLS proteins from different plants was constructed with Clustal X 2.0 and MEGA 6.0 software using the neighbor-joining (NJ) method (Tamura et al., 2013).

\section{Results}

\section{cDNA cloning of GbFLS2}

Leaves of G. biloba, which contain abundant secondary metabolites such as flavonols and flavonols, were used to clone GbFLS2. PCR was done with specific primers and the cDNA as template. The length of obtained GbFLS2 cDNA is $1251 \mathrm{bp}$, and the open reading frame is $1017 \mathrm{bp}$ encoding 338 amino acids. The 5 '-untranslated region and 3 '-untranslated region of GbFLS2 cDNA were $66 \mathrm{bp}$ and 168 bp, respectively (Fig. 1).

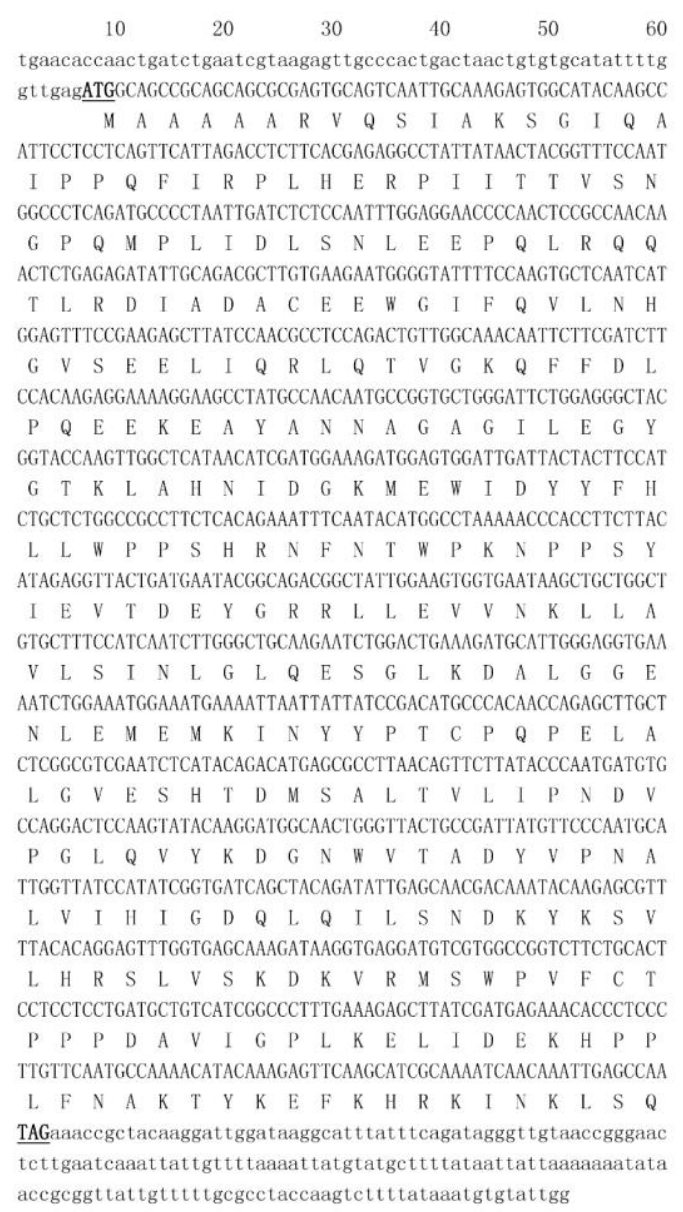

Fig. 1: cDNA sequence of GbFLS2 gene and its deduced amino acids. The start codon (ATG) and the stop (TAG) are underlined, noncoding regions are indicated in lowercase. 


\section{Characterization of the deduced GbFLS2 protein}

The putative GbFLS2 protein contains 338 amino acids. Computer $\mathrm{pI} / \mathrm{Mw}$ analysis showed the molecular weight and isoelectric point of GbFLS2 protein were $38.1 \mathrm{kDa}$ and 5.72, respectively. The secondary structure of GbFLS protein was predicted by using SOPMA tool. It was found that the percentages of alpha helix, extended strand, random coil and beta turn in the secondary structure were $37.57 \%, 18.64 \%, 35.80 \%$ and $7.99 \%$, respectively (Fig. 2).

\section{Homology analysis of GbFLS2 protein}

Homology analysis was completed with BLASTP (NCBI) and Align X (Vector NTI 11.5). The results showed the deduced GbFLS protein belongs to OG-Fe (II) dioxygenase superfamily, conserved DIOX_N and
2OG-FeII_oxy domains were found in the protein. 2OGFeII_oxy superfamily contains members of the 2oxoglutarate (2OG) and Fe (II)-dependent oxygenase superfamily. DIOX_N is the highly conserved $\mathrm{N}$ terminal region of proteins with 2-oxoglutarate/Fe (II)dependent dioxygenase activity. Sequence alignment found that the putative GbFLS2 was $67 \%$ identical to FLS1 from G. biloba (ACY00393), 61\% identical to FLS from Pinus radiata (AGY80773), 57\% identical to FLS from Dioscorea alata (AIY60790), and 56\% identical to FLS from Camellia sinensis (ABM88786), Epimedium sagittatum (ABY63659), Theobroma cacao (EOY09743), and Tricyrtis sp. Shinonome (BAU20368) (Fig. 3). The homologous sequence of FLS among different species indicated the FLS proteins might keep a conservative during the molecular evolution, and GbFLS2 was a member of the OG-Fe (II) dioxygenase superfamily.
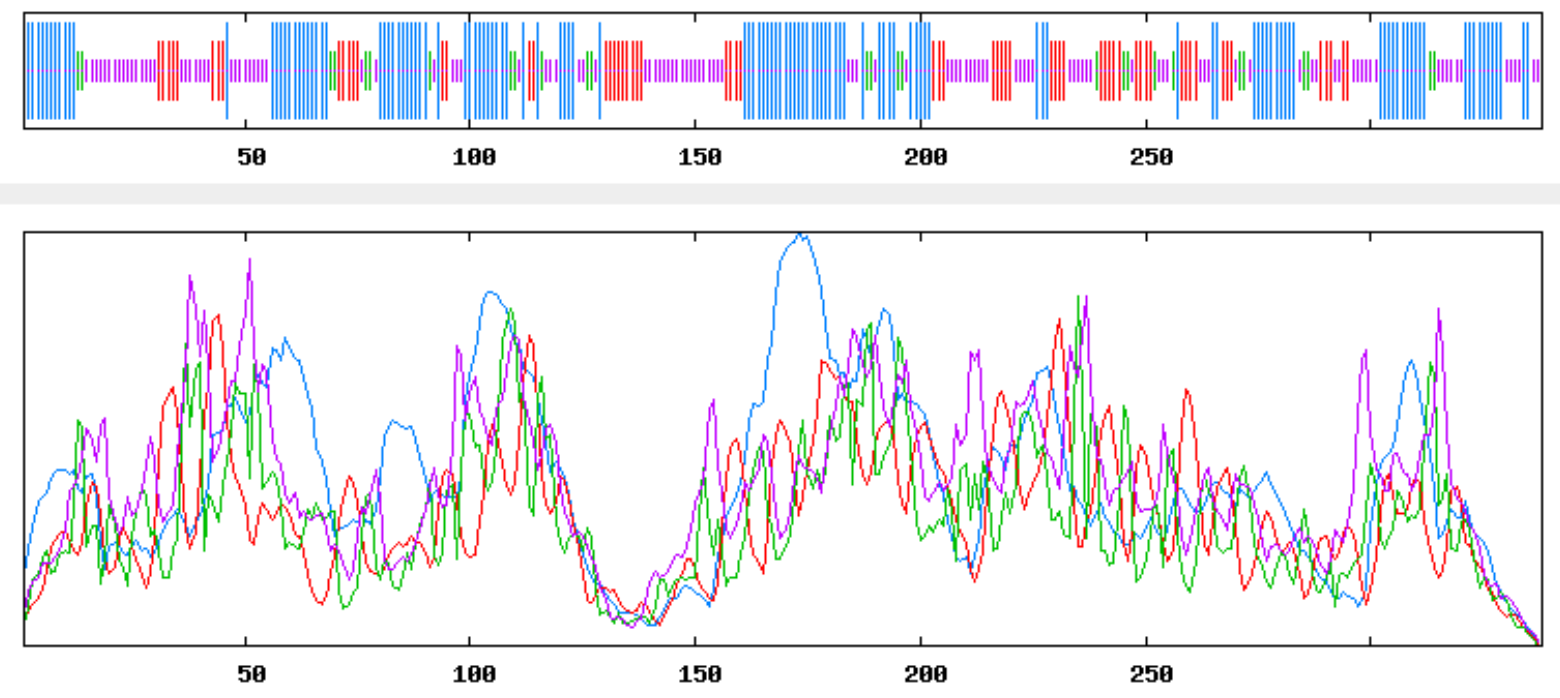

Fig. 2: The predicted secondary structures of GbFLS2 protein.

\section{Phylogenetic analysis of FLS proteins}

In order to analyze evolutionary relationships of FLS proteins among the various species. Using Clustal X 2.0 and MEGA 6.0 software, the phylogenetic tree was constructed by the neighbor-joining method. As shown in Fig. 4, FLS proteins were clustered into two group gymnosperm and angiosperm, and the putative GbFLS2 protein together with GbFLS1 and PrFLS (Pinus radiata) was clustered into the branch of gymnosperm plants. FLS proteins from closely related species shared the same subclades. FLS proteins from Rosaceae such as Rosa rugosa, Fragaria $\times$ ananassa, Malus domestica and Prunus persica were grouped into the same cluster, while FLS proteins of Arabidopsis thaliana and Brassica rapa that from Cruciferae were grouped into another cluster. The phylogenetic tree indicated that GbFLS shared a common evolutionary origin with the gymnosperm species FLS proteins.

\section{Discussion}

Flavonol Synthase, as a key enzyme involved in the flavonol biosynthetic pathway, has been studied in many plants (Li et al., 2013; Toh et al., 2013; Takahashi et al., 2007; Falcone Ferreyra et al., 2010; Xu et al., 2012; Mahajan et al., 2011). In plants, flavonol synthase could catalyse flavonoids synthesis. 


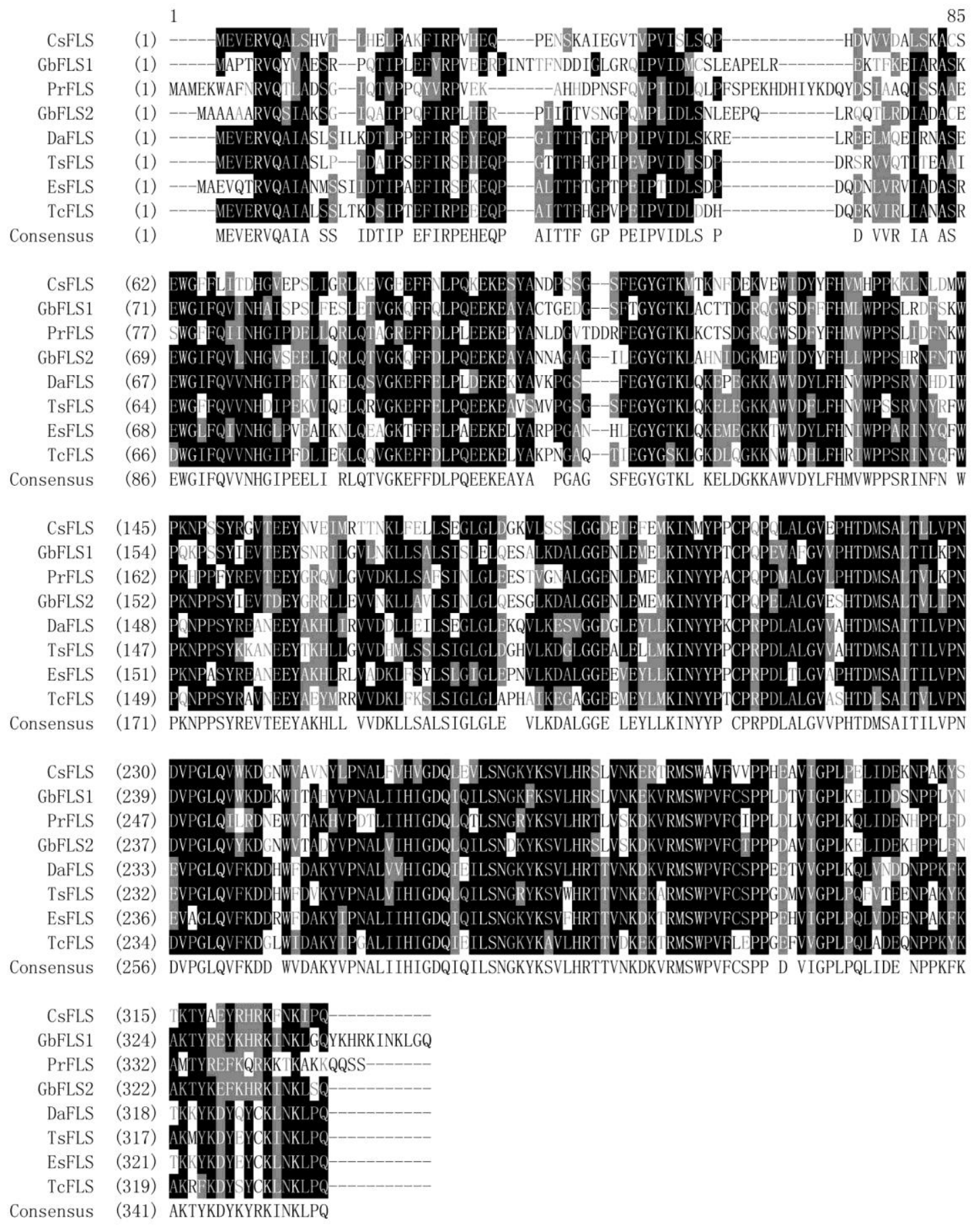

Fig. 3: Multiple sequence alignment of the deduced FLS with other proteins from other plants. The completely identical amino acids are indicated with black background. The conserved amino acids are indicated with grey background. Non-similar amino acids are indicated with white background.

In Acacia confusa, AcFLS could enzymatically transform dihydromyricetin, dihydroquercetin, and naringenin into the flavonols myricetin, quercetin, and kaempferol, respectively (Toh et al., 2013). In maize, ZmFLS1 was also able to convert DHK and DHQ to produce the flavonols $\mathrm{K}$ and $\mathrm{Q}$, though it fails to convert
Nar to DHK (Falcone Ferreyra et al., 2010). Flavonol synthase also could play an important role in the physiological activities of plants. In tartary buckwheat, different FtFLS isoforms of buckwheat have different functions in the response to environmental stress; transcription of FtFLS1 was inhibited by the exogenous 
application of $\mathrm{ABA}$, SA and $\mathrm{NaCl}$, while FtFLS2 was not affected by $\mathrm{ABA}$ but up-regulated by $\mathrm{SA}$ and $\mathrm{NaCl}$ (Li et al., 2013). In A. thaliana, 6 FLS isoforms were identified from $A$. thaliana that exhibited tissue or cell type-specific promoter activities. However, only
AtFLS1 was shown to play a primary role in flavonol biosynthesis in A. thaliana (Owens et al., 2008). In $G$. biloba, GbFLS1 is a bifunctional enzyme within the flavonol biosynthetic pathway, but function of other FLS isoforms is still unknown (Xu et al., 2012).

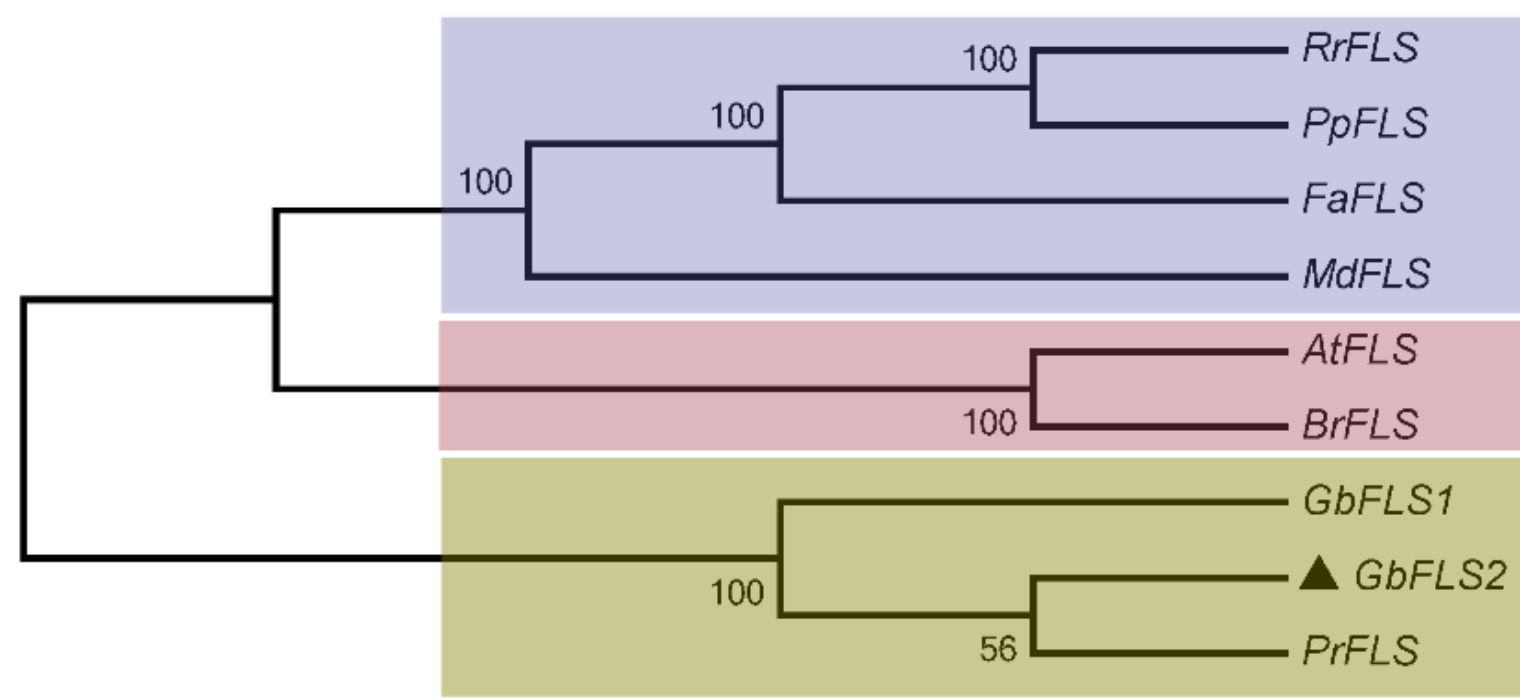

Fig. 4: Phylogenetic tree of FLS proteins from different species. The numbers at each node represent the bootstrap values (with 1,000 replicates). GenBank accession number are as follows: PrFLS, Pinus radiata, AGY80773; GbFLS2, Ginkgo biloba,ACY0039; AtFLS, Arabidopsis thaliana, AAB41504;BrFLS, Brassica rapa, XP_009122482;RrFLS, Rosa rugosa, AIS22436; FaFLS, Fragaria ×ananassa, AAZ78661; MdFLS, Malus domestica, AAD26261; PpFLS, Prunus persica, AJO70134.

In this study, one $F L S$ gene, GbFLS2, was isolated from G. biloba. The ORF of GbFLS2 was 1017 bp, encoding 338 amino acids. Homology analysis found that the deduced GbFLS2 belong to 2-ODD superfamily, and was high identity with FLS proteins from other plants. Phylogenetic analysis suggested that the flavonol Synthase of gymnosperm plants may be from the same ancestor. Above all, the cDNA sequence and protein structure of $G b F L S 2$ was similar to other $F L S$ genes that function had been identified. Therefore, we hypothesized that the GbFLS2 maybe also involved in regulation of flavonol biosynthesis, which need to be further studied. The isolation and structural analysis of GbFLS2 gene provided a foundation for further studying the function of FLS proteins in G. biloba.

\section{Conflict of interest statement}

Authors declare that they have no conflict of interest.

\section{Acknowledgement}

This work was supported by the National Natural Science Foundation of China (31500546), the Doctor
Foundation of Yangtze University (801190010127), and Yangtze University Undergraduate Training Programs for Innovation and Entrepreneurship (2016155).

\section{References}

Akbar Böhm, H., Boeing, H., Hempel, J., Raab, B., Kroke, A., 1998. Flavonols, flavone and anthocyanins as natural antioxidants of food and their possible role in the prevention of chronic diseases. Z. Ernahrungswiss. 37(2), 147-163.

Cheng, H., Li, L., Cheng, S., Cao, F., Wang, Y., Yuan, H., 2011. Molecular cloning and function assay of a chalcone isomerase gene ( $\mathrm{GbCHI})$ from Ginkgo biloba. Plant Cell Rep. 30(1), 49-62.

Falcone Ferreyra, M. L., Rius, S., Emiliani, J., Pourcel, L., Feller, A., Morohashi, K., Casati, P., Grotewold, E., 2010. Cloning and characterization of a UV-Binducible maize flavonol synthase. Plant J. 62(1), 77-91.

Havsteen, B. H., 2002. The biochemistry and medical significance of the flavonoids. Pharmacol. Therapeut. 96(2), 67-202.

Kim, J. D., Liu, L., Guo, W., Meydani, M., 2006. 
Chemical structure of flavonols in relation to modulation of angiogenesis and immune-endothelial cell adhesion. J. Nutr. Biochem. 17(3), 165-176.

Li, X., Kim, Y. B., Kim, Y., Zhao, S., Kim, H. H., Chung, E., Lee, J. H., Park, S. U., 2013. Differential stress-response expression of two flavonol synthase genes and accumulation of flavonols in tartary buckwheat. J. Plant Physiol. 170(18), 1630-1636.

Lin, G. Z., Lian, Y. J., Ryu, J. H., Sung, M. K., Park, J. S., Park, H. J., Park, B. K.,Shin, J. S., Lee, M. S., Cheon, C. I., 2007. Expression and purification of His-tagged flavonol synthase of Camellia sinensis from Escherichia coli. Protein Expr. Purif. 55(2), 287-292.

Mahajan, M., Ahuja, P. S., Yadav, S. K., 2011. Posttranscriptional silencing of flavonol synthase mRNA in tobacco leads to fruits with arrested seed set. PLoS One. 6(12), e28315.

Owens, D. K., Alerding, A. B., Crosby, K. C., Bandara, A. B., Westwood, J. H., Winkel, B. S. J., 2008. Functional analysis of a predicted flavonol synthase gene family in Arabidopsis. Plant Physiol. 147(3), 1046-1061.

Pang, Y., Shen, G., Wu, W., Liu, X., Lin, J., Tan, F., Sun, X., Tang, K., 2005. Characterization and expression of chalcone synthase gene from Ginkgo biloba. Plant Sci. 168(6), 1525-1531.

Shen, G., Pang, Y., Wu, W., Deng, Z., Zhao, L., Cao, Y., Sun, X., Tang, K., 2006. Cloning and characterization of a flavanone 3-hydroxylase gene from Ginkgo biloba. Biosci. Rep. 26(1), 19-29.
Takahashi, R., Githiri, S. M., Hatayama, K., Dubouzet, E. G., Shimada, N., Aoki, T., Ayabe, S. I., Iwashina, T., Toda, K., Matsumura, H., 2007. A single-base deletion in soybean flavonol synthase gene is associated with magenta flower color. Plant Mol. Biol. 63(1), 125-135.

Tamura, K., Stecher, G., Peterson, D., Filipski, A., Kumar, S., 2013. MEGA6: Molecular evolutionary genetics analysis version 6.0. Mol. Biol. Evol. 30(12), 2725-2729.

Toh, H. C., Wang, S. Y., Chang, S. T., Chu, F. H., 2013. Molecular cloning and characterization of flavonol synthase in Acacia confusa. Tree Genet. Genomes. 9(1), 85-92.

Wellmann, F., Lukačin, R., Moriguchi, T., Britsch, L., Schiltz, E., Matern, U., 2002. Functional expression and mutational analysis of flavonol synthase from Citrus unshiu. Eur. J. Biochem. 269(16), 4134-4142.

Xu, F., Cai, R., Cheng, S., Du, H., Wang, Y., Cheng, S., 2008. Molecular cloning, characterization and expression of phenylalanine ammonia-lyase gene from Ginkgo biloba. Afr. J. Biotechnol. 7(6), 721729.

Xu, F., Cheng, S. Y., Cheng, S. H., Wang, Y., Du, H. W., 2007. Time course of expression of chalcone synthase gene in Ginkgo biloba. J. Plant Physiol. Mol. Biol. 33(4), 309-317.

Xu, F., Li, L., Zhang, W., Cheng, H., Sun, N., Cheng, S., Wang, Y., 2012. Isolation, characterization, and function analysis of a flavonol synthase gene from Ginkgo biloba. Mol. Biol. Rep. 39(3), 2285-2296.

\section{How to cite this article:}

Zeng, H., Yang, L., Rong, H., Zhang, W., Xu, F., Zhang, X., 2017. Cloning and sequence analysis of a Flavonol Synthase (FLS2) gene from Ginkgo biloba. Int. J. Curr. Res. Biosci. Plant Biol. 4(4), 14-19.

doi: https://doi.org/10.20546/ijcrbp.2017.404.003 